Short Note

\title{
Prevalence of Human Norovirus by Genotype in Contaminated Groundwater in Korea Over the Last Decade (2007-2016)
}

\section{Siwon Lee, Sang-Jung Park, Ji-Hye Kim, Wonseok Lee, Hyen-Mi Chung*}

Environmental Infrastructure Research Department, National Institute of Environmental Research, Incheon 22689, Korea

\section{*Corresponding author:}

Tel: +82-32-560-8300, Fax: +82-32-563-7085, E-mail: hyenmic@korea.kr

\begin{abstract}
This study investigated the presence of human Norovirus (HuNoV) by genotype in 1,486 groundwater samples, collected from 843 groundwater wells suspected of contamination during 2007-2016, in Republic of Korea. We identified and genotyped 186 HuNoV sequences in 178 HuNoV-positive samples using the RIVM-NoroNet norovirus genotyping tool (NGT) and phylogenetic tree analysis based on RIVM-NoroNet reference sequences. HuNoV GII was more prevalent than GI. The major genotypes detected were HuNoV GII.4 (43.0\%), GII.22 (15.6\%), GI.5 (10.2\%) and GI.1 (8.6\%); 14 genotypes accounted for $<5.0 \%$. Increasing HuNoV
\end{abstract}


GII.17 has displayed a worldwide trend, including in Europe and Asia since 2010, and it was the dominant HuNoV genotype during 2013-2014 in Korea. However, HuNoV GII.4 was the major genotype detected in groundwater in Korea in 2015-2016, and it $\dot{\mathbf{s}}$ becoming increasingly prevalent similar to HuNoV GII.17.

Keywords: genotype; groundwater; human norovirus; HuNoV; HuNoV genotype

\section{Introduction}

Human Norovirus (HuNoV) is a member of the family Caliciviridae, which can be subdivided into more than 32 genetic clusters [1]. HuNoV causes viral gastroenteritis in humans of all ages and shows seasonality, being especially prevalent during the winter [2]. HuNoV can infect humans through contaminated water or food via the fecal-oral route [3], and it is possible to re-infect individuals using the clinical samples of patients who were infected in rivers [4]. The first outbreak of HuNoV was reported in the US (Norwalk, Ohio) [5], and the virus has since been observed worldwide, including in Asia. The first official HuNoV outbreak from groundwater was reported in 2004 in Jeju Island, Korea [6]. Most human HuNoV infections due to groundwater were of genotypes GI and GII, and groundwater monitoring is critical to prevent outbreaks [7]. Nationwide $\mathrm{HuNoV}$ monitoring has been performed during 2007 to 2016 in National Institute of Environmental Research, Ministry of Environment, Republic of Korea focusing on groundwater wells suspected as being contaminated by relevant organizations, measurement of groundwater quality, and consultation meetings.

The HuNoV GI and GII detection methods that have been used in Korea include RNA extraction and conventional nested polymerase chain reaction (PCR). Specifically, HuNoVspecific bands were analyzed using gel-electrophoresis, followed by nucleotide sequence 
analysis. The basic local alignment search tool (BLAST) of the National Center for Biotechnology Information (NCBI), and phylogenetic tree analysis based on HuNoV reference sequences from NCBI designated independently in Korea have been used to detect similarities among virus samples and to perform detailed genotyping of HuNoVs [8]. The norovirus genotyping tool (NGT) of RIVM-NoroNet has been used worldwide to detect HuNoV genotypes; recently, it was used in Korea to detect HuNoVs in the environment, in clinical samples, and in food (fish and shellfish). This study investigated the presence of HuNoV in groundwater (by genotype) over the last decade in Korea by using NGT and phylogenetic treebased analyses based on RIVM-NoroNet reference sequences.

Water sampling and nucleic acid analysis of groundwater were performed as described previously $[7,8]$. Nucleic acid was extracted from $140 \mu \mathrm{l}$ of concentrated solution using the QIAamp Viral RNA Mini Kit (Qiagen, Hilden, Germany) according to the manufacturer's protocol. For gene amplification of HuNoV GI and GII, reverse transcription (RT)-PCR and nested PCR were used to target the open reading frame ORF1-ORF2 junction sequences. The amplicon of HuNoV GI was 330 base-pairs long (bp) in the RT-PCR analysis and 315 bp long in the nested PCR analysis, whereas the amplicons of HuNoV GII were 341 and 314 bp long, respectively [9]. RT-PCR amplification was performed using the One Step RT-PCR kit (Qiagen) in a total volume of $20 \mu 1$, including $5 \mu 1$ RNA templates. RT was conducted at $45^{\circ} \mathrm{C}$ for 30 min; then, PCR was performed by denaturation at $94^{\circ} \mathrm{C}$ for $30 \mathrm{~s}$, followed by 35 cycles of $94^{\circ} \mathrm{C}$ for $30 \mathrm{~s}, 55^{\circ} \mathrm{C}$ for $30 \mathrm{~s}, 72^{\circ} \mathrm{C}$ for $90 \mathrm{~s}$, and a final extension at $75^{\circ} \mathrm{C}$ for $7 \mathrm{~min}$. Nested PCR was performed using the EmeraldAmp ${ }^{\circledR}$ PCR Master Mix (Takara, Japan) in a reaction containing $25 \mu 1$ EmeraldAmp ${ }^{\circledR}$ PCR Master Mix, $2 \mu 1$ each of 20 pmol primers (forward and reverse), 2 $\mu 1$ RT-PCR product, and $19 \mu \mathrm{l}$ nucleic acid-free water. Nested PCR was performed using the same PCR conditions described above $[9,10]$. HuNoV GI and GII positive controls developed in Korea were used as described previously [10]. Sequencing of specific bands of HuNoV GI 
and GII was performed by Macrogen Co. Ltd. (Seoul, Korea) and the analyzed sequences were verified. In addition, as a homogeneous sequence is required for HuNoV genotyping, gene cloning was performed [8] when two or more genotypes were suspected to be present. The RIVM-NoroNet NGT was used for HuNoV genotype analysis, and phylogenetic trees were constructed based on RIVM-NoroNet HuNoV GI and GII references. Multiple sequence alignment and phylogenetic trees were constructed using BioEdit (ver. 7.1.3) [11] and MEGA software (ver. 6.06) [12]. The neighbor-joining (NJ) method with Jukes-Cantor and P-distances was used to generate phylogenetic trees and distance models. A total of 1,486 samples were collected from 843 contaminated groundwater wells during 2007-2016 (Table 1); 178 samples (approximately $12.0 \%$ ) were positive for $\mathrm{HuNoV}$, and two $\mathrm{HuNoV}$ genotypes were found to be present in 8 of the samples. However, this positivity rate may not be informative regarding overall $\mathrm{HuNoV}$ prevalence because we focused only on groundwater wells suspected of being contaminated rather than performing random sampling. However, the trend of HuNoV genotype and detection ratios were significant according to the year, and 186 sequences were analyzed from $178 \mathrm{HuNoV-positive} \mathrm{samples,} \mathrm{including} 10$ shorter sequences (65-72 bp). Therefore, a total of 186 sequences were analyzed in the investigation of HuNoV prevalence using the NGT, and 10 shorter sequences (65-72 bp; data not shown) were analyzed to determine the presence of the HuNoV GI and GII genotypes. Phylogenetic tree analysis based on RIVM-NoroNet reference sequences was conducted on 10 sequences, and a detailed genotypic analysis was performed (Supplementary Fig. S1).

HuNoV GI and GII were detected in 176 groundwater samples; GI was found in 49 samples $(27.8 \%)$, GII in 118 samples $(67.1 \%)$, and both GI and GII in 9 samples $(5.1 \%)$. The ratio of HuNoV GI to GII was approximately 3:7, indicating that GII was detected $\sim 2.3$ times more frequently than GI (data not shown). Nine samples contained both GI and GII. The most frequently detected HuNoV genotype in groundwater samples collected over the last decade was 
GII.4 (in 80 samples, 43.0\%), followed by GII.22 (29 samples, 15.6\%), GI.5 (19 samples, 10.2\%) and GI.1 (16 samples, 8.6\%); genotypes accounting for $<5.0 \%$ of all HuNoVs included GII.17 (9 samples, 4.8\%), GI.6 (9 samples, 4.8\%), GI.4 (6 samples, 3.2\%), GII.6 (4 samples, 2.2\%), GI.8 (3 samples, 1.6\%), GII.3 (2 samples, 1.1\%), GII.13 (2 samples, 1.1\%), GI.2 (1 sample, 0.5\%), GI.9 (1 sample, 0.5\%), GII.1 (1 sample, 0.5\%), and GII.8 (1 sample, 0.5\%). One sample contained both GI.3 and GI.7, and both were included in the overall count (Table 2). Therefore, 18 different $\mathrm{HuNoV}$ genotypes were detected in Korean groundwater samples collected between 2007 and 2016 (GI.1-9 and GII.1, 3, 4, 6, 8, 10, 13, 17, and 22), whereas 13 HuNoV GI and GII genotypes (GII.2, 5, 7, 9, 11, 12, 14, 15, 16, 18, 19, 20, and 21) were not found (Table 2).

The annual prevalence trends for HuNoV genotypes over the last 10 years are shown in Table 2 and Figure 1. The GI.6 and GII.22 genotypes were detected in the preliminary survey period in 2007, while 13 other genotypes (GI.1-9, and GII.1, 4, 13, and 22) were identified in 2008 when the nationwide investigation began in earnest. The number of HuNoV genotypes detected decreased to five in 2009 (GI.5 and 6, and GII.3, 4, and 22) and 2010 (GI.5, and GII.4, 6, 17, and 22). During 2011-2012, no HuNoV GI and GII genotypes were detected in samples at any sampling point. A single genotype, GII.17, was detected in 2013-2014, corresponding to the global trend about increasing HuNoV GII.17, including Europe and Asia, which showed a trend from 2010, while GII.4 was the only HuNoV genotype identified in 2015. HuNoVs GII.17 and GII.4 both were detected at 50\% in 2016.

HuNoV GII.4 was the most infectious HuNoV genotype in the last decade worldwide. However, the rate of GII.17 infection increased by $\sim 82 \%$ from 2014 to 2015 in Guangdong, China [13], with similar reports in the US and Europe, including Italy and sporadic cases in Romania [14-16]. A recent report described the emergence of GII.17, suggesting the possibility of latent activity [17]. In Korea, HuNoV GII.4 was detected most frequently during 2007-2009, 
with the rate showing a decrease in 2010 in contrast with increased rates of infection by the GI.5, GII.6, and GII.17 genotypes. According to global trends, it was expected that HuNoV GII.17 would be detected more consistently than GII.4 in Korea. While HuNoV GII.17 was detected during 2013-2014, similar to the results for China, the US and Europe, HuNoV GII.4 was detected in 2015 [13-17], and was also detected along with HuNoV GII.17 in 2016 (Table 2 and Fig. 2). Over the last decade, HuNoV detection rates have decreased due to improved groundwater monitoring and management of HuNoVs in the environment, as well as in patients and food (fish and shellfish); moreover, the range of HuNoV genotypes detected seems to be becoming less diverse. Nevertheless, HuNoVs GII.4 and GII.17 are still being detected, and it will be necessary to manage $\mathrm{HuNoV}$ cases according to genotype in the future.

The concentrations of HuNoV in the environment, including groundwater, are lower than those in clinical samples from patients or food; therefore, collecting large numbers of samples from the environment and detecting virus concentrations therein requires laborintensive monitoring efforts. Moreover, management of groundwater is difficult and costly on a nationwide scale. Accordingly, continuous efforts are required. Currently, HuNoV genotypes in Korea are monitored in various samples, including those from patients, food, marine products [18-23], and groundwater near agricultural and stockbreeding regions [29]. For greater understanding, prevention, and management of HuNoVs, further studies of a variety of sample types are needed using the genotyping method described herein. 


\section{Conclusions}

HuNoV GIIs were detected at higher rates than HuNoV GIs in contaminated groundwater samples collected in Korea over the last decade. The most common HuNoV genotype was GII.4 ( 43.0\%), followed by GII.22 (15.6\%), GI.5 (10.2\%), and GI.1 (8.6\%). The genotypes accounting for $<5.0 \%$ of all HuNoVs were as follows (in decreasing order): GI.6, GII.17, GI.4, GII.6, GI.8, GII.3, GII.13, GI.3, GI.7, GI.2, GI.9, GII.1, GII.8, and GII.10. Recently, the prevalence of HuNoVs and number of genotypes detected has decreased. HuNoV GII.4 was the most frequently detected HuNoV genotype in Korea until 2009. HuNoV GII.17 showed an increased prevalence rate in Korea from 2010, corresponding to trends observed in China, Europe and the US; HuNoV GII.17 became the major HuNoV genotype detected in groundwater in Korea between 2013 and 2014. However, HuNoV GII.4 was again the dominant HuNoV genotype in 2015, and was detected at the same rate as GII.17 in 2016. Therefore, HuNoV GII.4 and GII.17 are the major HuNoV genotypes in groundwater in Koreas. Further monitoring studies are needed, using the genotyping methods described herein, for management of future $\mathrm{HuNoV}$ outbreaks.

\section{Acknowledgment}

The present research was conducted by a grant from the National Institute of Environmental Research, Ministry of Environment, Republic of Korea. 


\section{References}

1. Vainio, K.; Myrmel, M. Molecular epidemiology of norovirus outbreak in Norway during 2000 to 2005 and comparision of four norovirus real-time reverse transcriptase PCR assays. J. Clin. Microbiol. 2006, 44, 3695-3702, doi: 10.1128/JCM.00023-06.

2. Lee, G.C.; Kim, M.J.; Kim, J.I.; Lee, C.H. Occurrence and molecular characterization of noroviruses in Korean surface water between 2007 and 2010. J. Microbiol. Biotechnol. 2014, 24, 556-562, http://dx.doi.org/10.4014/jmb.1311.11089.

3. Morillo, S.G.; Timenetsky, M.C.S.T. Norovirus: an overview. Rev. Assoc. Med. Bras. 2011, 57, 453-458, http://dx.doi.org/10.1590/S0104-42302011000400023.

4. Lee, G.C.; Jheong, W.H.; Jung, G.S.; Oh, S.A.; Kim, M.J.; Rhee, O.J.; Park, S.; Lee, C.H. Detection and molecular characterization of human noroviruses in Korean groundwater between 2008 and 2010. Food Environ. Virol. 2012, 4, 115-123, doi: 10.1007/s12560-0129084-y.

5. Glass, R.I.; Parashar, U.D.; Estes, M.K. Norovirus gastroenteritis. N. Engl. J. Med. 2009, 361, 1176-1785, doi: 10.1056/NEJMra0804575.

6. Kim, S.H.; Cheon, D.S.; Kim, J.H.; Lee, D.H.; Jheong, W.H.; Heo, Y.J.; Chung, H.M.; Jee, Y.M.; Lee, J.S. Outbreaks of gastroentertis that occurred during school excursions in Korea were associated with several waterboone strains of norovirus. J. Clin. Microbiol. 2005, 43, 4836-4839, doi: 10.1128/JCM.43.9.4836-4839.2005.

7. National Institute Environmental Research. Manual for Norovirus management in groundwater. 2010. 11-1480523-000616-01.

8. National Institute Environmental Research. Investigation on Norovirus contamination in groundwater (III). 2010. http://webbook.me.go.kr/DLi-File/NIER/06/008/5503844.pdf

9. Lee, B.R.; Lee, S.G.; Park, J.H.; Kim, K.Y.; Ryu, S.R.; Rhee, O.J.; Park, J.W.; Lee, J.S.; Paik, 
S.Y. Norovirus contamination levels in ground water treatment systems used for foodcatering facilities in South Korea. Viruses. 2013, 2, 1646-1654, doi: 10.3390/v5071646.

10. Lee, S.G.; Lee, S.H.; Park, S.W.; Suh, C.I.; Jheong, W.H.; Oh, S.; Paik, S.Y. Standardized positive controls for detection of norovirus by reverse transcription PCR. Virol. J. 2011, 260, 1-8, doi: 10.1186/1743-422X-8-260.

11. Hall, T.A. BioEdit: a user-friendly biological sequence alignment editor and analysis program for Windows 95/98/NT. Nucleic Acids Symp. Ser. 1999, 41, 95-98, doi:10.1021/bk-1999-0734.ch008.

12. Tamura, K.; Stecher, G.; Peterson, D.; Filipski, A.; Kumar, S. MEGA6: molecular evolutionary genetics analysis version 6.0. Mol. Biol. Evol. 2013, 30, 2725-2729, doi: 10.1093/molbev/mst197.

13. Lu, J.; Sun, L.; Fang, L.; Yang, F.; Mo, Y.; Lao, J.; Zheng, H.; Tan, X.; Lin, H.. Rutherford, S.; Guo, L.; Ke, C.; Hui, L. Gastroenteritis outbreaks caused by Norovirus GII.17, Guangdong province, China, 2014-2015. Emerg. Infect Dis. 2015, 21, 1240-1242, doi: 10.3201/eid2107.150226.

14. Parra, G.I.; Green, K.Y. Genome of emerging norovirus GII.17, United States, 2014. Emerg. Infect Dis. 2015, 21, 1477-1479, doi: 10.3201/eid2108.150652.

15. Medici, M.C.; Tummolo, F.; Calderaro, A.; Chironna, M.; Giammanco, G.M.; De Grazia, S.; Arcangeletti, M.C.; De Conto, F.; Chezzi, C.; Martella, V. Identification of the novel Kawasaki 2014 GII.17 human norovirus strain in Italy, 2015. Euro Surveill. 2015, 20, 30010, doi: 10.2807/1560-7917.

16. Dinu, S.; Nagy, M.; Negru, D.G.; Popovici, E.D.; Zota, L.; Oprișan, G. Molecular identification of emergent GII.P17-GII.17 norovirus genotype, Romania, 2015. Euro Surveill. 2016, 21, 30141, doi: 10.2807/1560-7917.

17. de Graaf, M.; van Beek, J.; Vennema, H.; Podkolzin, A.T.; Hewitt, J.; Bucardo, F.; 
Templeton, K.; Mans, J.; Nordgren, J.; Reuter, G.; Lynch, M.; Rasmussen, L.D.; Iritani, N.; Chan, M.C.; Martella, V.; Ambert-Balay, K.; Vinjé, J.; White, P.A.; Koopmans, M.P. Emergence of a novel GII.17 norovirus - End of the GII.4 era?. Euro Surveill. 2015, 20, 21178. doi: http://dx.doi.org/10.2807/1560-7917.ES2015.20.26.21178.

18. Song, J.H.; Sun, D.S.; Park, J.S.; Choi, Y.J.; Nam, H.S.; Kim, Y.B.; Jung, E.H.; Baek, K.A.; Park, K.S. Comparison of clinical features of Norovirus and Rotavirus gastroenteritis in hospitalized children and Norovirus genotype analysis. Infect. Chemother. 2010, 42, 143 148. doi: $10.3947 /$ ic.2010.42.3.143.

19. Thoi, T.C. Evolutionary phylodynamics of noroviruses circulating in Republic of Korea between 2011 and 2012. Master Thesis, Chungang University, Seoul, August 2013.

20. Gong, Y.W.; Oh, B.Y.; Kim, H.Y.; Lee, M.Y.; Kim, Y.H.; Go, J.M.; Lee, J.M.; Jheong, H.S.; Cheon, D.S. Molecular epidemiologic investigation of Norovirus infections in Incheon city, Korea, from 2005 to 2007. J. Bacteriol. Virol. 2008, 38, 249-257. https://synapse.koreamed.org/pdf/10.4167/jbv.2008.38.4.249.

21. Kim, N.H.; Min, S.K.; Park, E.H.; Park, Y.K.; Jin, S.H.; Ryu, B.S. Study on gastroenteric Norovirus genotypes in Busan, Korea, from 2008 to 2010. Ann. Rep. Busan Metropolitan City Institute of Health and Environ. 2010, 20, 9-17.

22. Ki, H.Y. Prevalence and genotypes of enteric viruses from children with diarrhea in Gwangju metropolitan city, 2008-2012. Ph.D. Thesis, Chonnam National University, Gwangju, February 2015.

23. Cho, H.G. Molecular epidemiology of noroviruses in Gyeonggi and development of improved TaqMan assay. Ph.D. Thesis, University of Seoul, Seoul, February 2012. 


\section{Figure Legend}

Fig. 1. The annual prevalence trends for HuNoV GI and GII genotypes over the last 10 years (2007-2016)

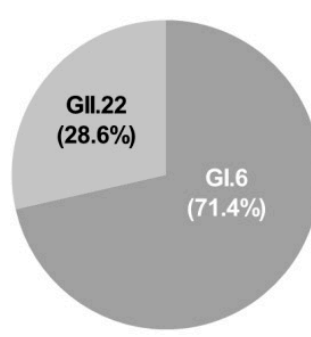

2007

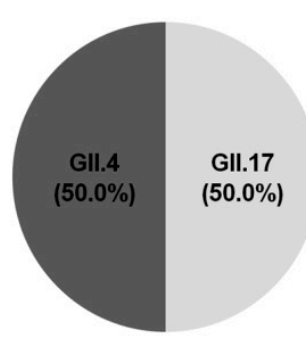

2016
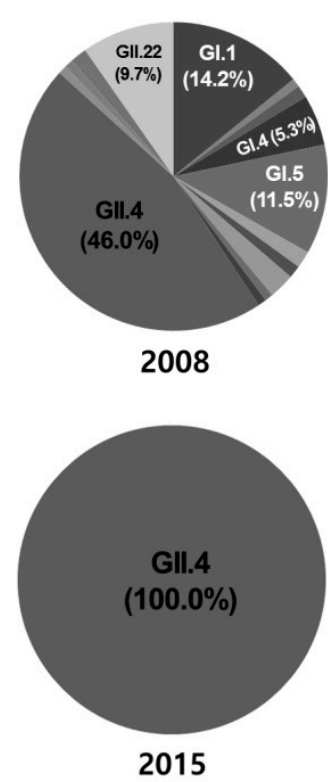

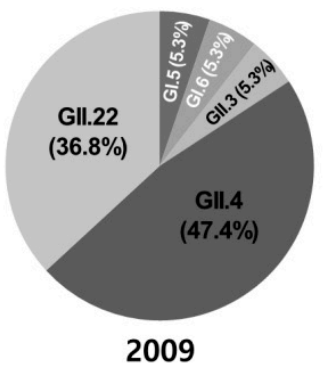

GII.17 (100.0\%)

2013-2014

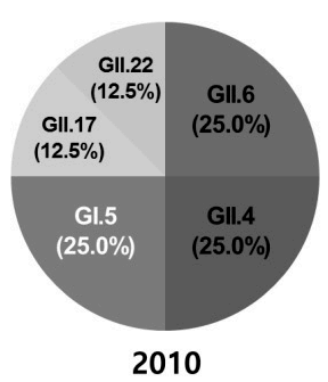

2011-2012 
Table 1. Investigations of human Norovirus (HuNoV) in groundwater performed in Korea over the last decade

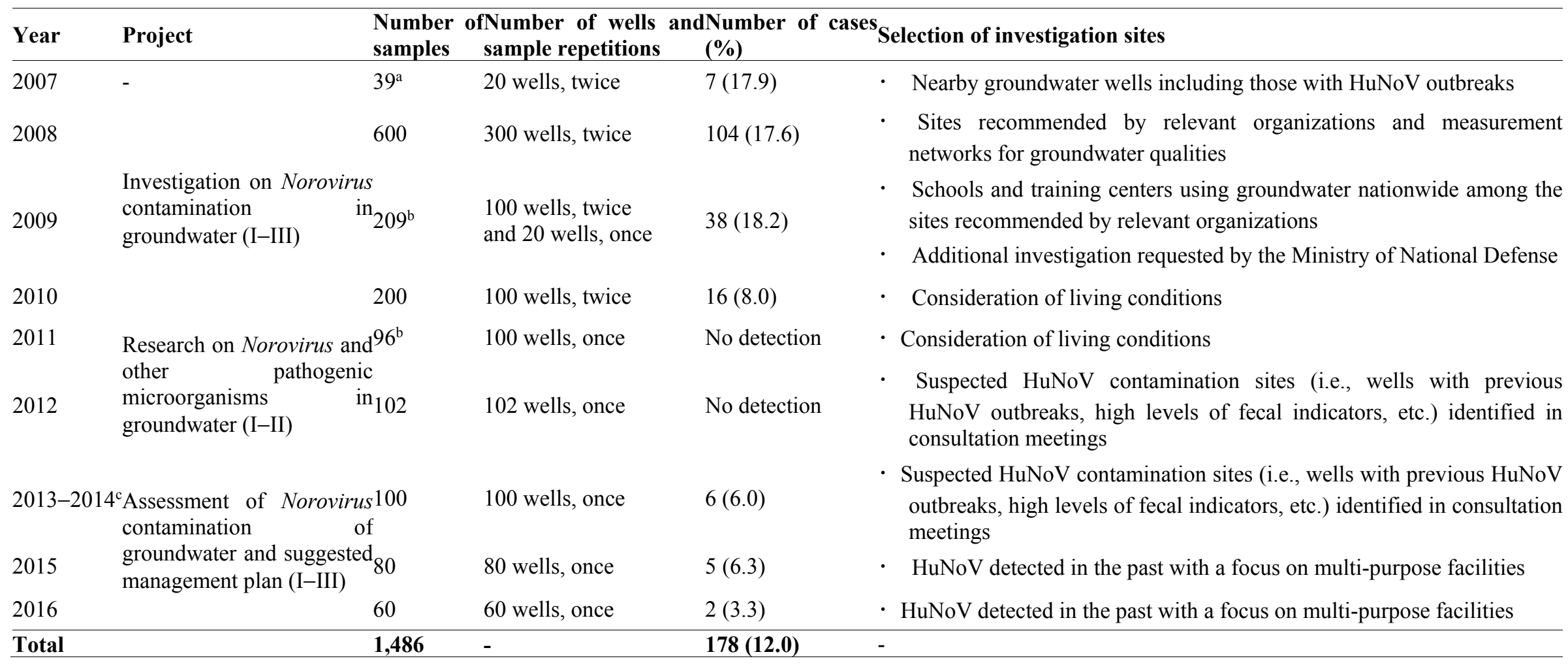

${ }^{\text {a } O n e ~ s a m p l e ~ c o u l d ~ n o t ~ b e ~ c o l l e c t e d ~ d u e ~ t o ~ a ~ c l o s e d ~ w e l l . ~}$

${ }^{b}$ For closed wells, nearby wells were selected; unsuitable wells (i.e., user absence, disavowal, etc.) were excluded from the analysis.

${ }^{c}$ Wells that required investigations during winter and spring (conducted from December 2013 to April 2014). 
Table 2. HuNoV genotyping using the RIVM-NoroNet norovirus genotyping tool (NGT) and phylogenetic tree analysis based on RIVM-NoroNet reference sequences.

\begin{tabular}{|c|c|c|c|c|c|c|c|c|c|}
\hline \multirow{2}{*}{\multicolumn{2}{|c|}{ Genotype }} & \multicolumn{7}{|l|}{ Year } & \multirow{2}{*}{-Total } \\
\hline & & 2007 & 2008 & 2009 & 2010 & 2013-2014 & 2015 & 2016 & \\
\hline \multirow{9}{*}{ GI } & 1 & - & 16 & - & - & - & - & - & 16 \\
\hline & 2 & - & 1 & - & - & - & - & - & 1 \\
\hline & 3 & - & $1^{\mathrm{a}}$ & - & - & - & - & - & $1^{\mathrm{a}}$ \\
\hline & 4 & - & 6 & - & - & - & - & - & 6 \\
\hline & 5 & - & 13 & 2 & 4 & - & - & - & 19 \\
\hline & 6 & 5 & 2 & 2 & - & - & - & - & 9 \\
\hline & 7 & - & $1^{\mathrm{a}}$ & - & - & - & - & - & $1^{\mathrm{a}}$ \\
\hline & 8 & - & 3 & - & - & - & - & - & 3 \\
\hline & 9 & - & 1 & - & - & - & - & - & 1 \\
\hline \multirow{22}{*}{ GII } & 1 & - & 1 & - & - & - & - & - & 1 \\
\hline & 2 & - & - & - & - & - & - & - & - \\
\hline & 3 & - & - & 2 & - & - & - & - & 2 \\
\hline & 4 & - & 52 & 18 & 4 & - & 5 & 1 & 80 \\
\hline & 5 & - & - & - & - & - & - & - & - \\
\hline & 6 & - & - & - & 4 & - & - & - & 4 \\
\hline & 7 & - & - & - & - & - & - & - & - \\
\hline & 8 & - & 1 & - & - & - & - & - & 1 \\
\hline & 9 & - & - & - & - & - & - & - & - \\
\hline & 10 & - & 1 & - & - & - & - & - & 1 \\
\hline & 11 & - & - & - & - & - & - & - & - \\
\hline & 12 & - & - & - & - & - & - & - & - \\
\hline & 13 & - & 2 & - & - & - & - & - & 2 \\
\hline & 14 & - & - & - & - & - & - & - & - \\
\hline & 15 & - & - & - & - & - & - & - & - \\
\hline & 16 & - & - & - & - & - & - & - & - \\
\hline & 17 & - & - & - & 2 & 6 & - & 1 & 9 \\
\hline & 18 & - & - & - & - & - & - & - & - \\
\hline & 19 & - & - & - & - & - & - & - & - \\
\hline & 20 & - & - & - & - & - & - & - & - \\
\hline & 21 & - & - & - & - & - & - & - & - \\
\hline & 22 & 2 & 11 & 14 & 2 & - & - & - & 29 \\
\hline \multicolumn{2}{|c|}{ Total } & 7 & $112^{\mathrm{b}}$ & 38 & 16 & 6 & 5 & 2 & $186^{\mathrm{b}}$ \\
\hline
\end{tabular}

a One sample (collected in 2008) contained both GI.3 and GI.7 based on NGT and phylogenetic tree analysis; both were included in the overall count.

${ }^{\mathrm{b}}$ Two genotypes were found to be present in eight samples. 